\title{
Caracterização clínico-epidemiológica da comorbidade tuberculose/diabetes mellitus: revisão integrativa
}

\author{
Clinical-epidemiological characterization of tuberculosis / diabetes comorbidity: integrative review \\ Caracterización clínico-epidemiológica de la comorbilidad tuberculosis / diabetes: revisión integradora
}

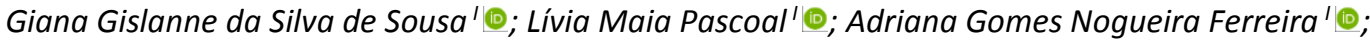 \\ Isaura Leticia Tavares Palmeira Rolim" "๑; Lívia Fernanda Siqueira Santos '®; Marcelino Santos Neto'® \\ 'Universidade Federal do Maranhão, Imperatriz, MA, Brasil; "Universidade Federal do Maranhão, São Luis, MA, Brasil
}

\begin{abstract}
RESUMO
Objetivo: sintetizar as evidências científicas acerca de características sociodemográficas, indicadores epidemiológicos de morbidade e manifestações clínicas dos casos associados de tuberculose e diabetes mellitus. Método: revisão integrativa da literatura com buscas realizadas nas bases Scopus, Literatura Latino Americana e do Caribe em Ciências da Saúde, Medical Literature Analysis and Retrieval System Online e nos portais National Library of Medicine, Biblioteca Virtual em Saúde Brasil e Scientific Eletronic Library Online, sendo a amostra final foi composta por dezesseis estudos. Resultados: observou-se que a alta prevalência de diabetes contribui para o aumento dos casos de tuberculose. Os pacientes com a comorbidade tendem a apresentar idade avançada, serem do sexo feminino e possuírem renda familiar mais baixa. $O$ diabetes afeta negativamente a gravidade da tuberculose pulmonar, provocando sintomas mais graves. Conclusão: as evidências apontadas têm potencial para guiar as intervenções em saúde e possibilitam a implementação de ações de saúde mais efetivas.

Descritores: Epidemiologia; Tuberculose; Diabetes Mellitus; Comorbidade.
\end{abstract}

\section{ABSTRACT}

Objective: to summarize the scientific evidence from sociodemographic characteristics, epidemiological indicators of morbidity, and clinical manifestations of cases of tuberculosis associated with diabetes mellitus. Method: this integrative review searched for literature in the Scopus, Latin American and Caribbean Health Sciences Literature, and Medical Literature Analysis and Retrieval System Online databases, and in the National Library of Medicine, Virtual Health Library Brazil and Scientific Electronic Library Online portals. The final sample comprised of sixteen studies. Results: the high prevalence of diabetes was found to contribute to the increase in tuberculosis cases. Patients with comorbidity tended to be older, female and with lower family income. Diabetes affects the severity of pulmonary tuberculosis adversely, causing more severe symptoms. Conclusion: the evidence found has the potential to guide health interventions and enable more effective health actions to be implemented. Descriptors: Epidemiology; Tuberculosis; Diabetes Mellitus; Comorbidity.

\section{RESUMEN}

Objetivo: resumir la evidencia científica a partir de características sociodemográficas, indicadores epidemiológicos de morbilidad y manifestaciones clínicas de los casos de tuberculosis asociados a diabetes mellitus. Método: esta revisión integradora buscó literatura en las bases de datos Scopus, Latin American and Caribbean Health Sciences Literature y Medical Literature Analysis and Retrieval System Online, y en los portales National Library of Medicine, Virtual Health Library Brasil y Scientific Electronic Library Online. La muestra final estuvo compuesta por dieciséis estudios. Resultados: se encontró que la alta prevalencia de diabetes contribuyó al aumento de casos de tuberculosis. Los pacientes con comorbilidad tendían a ser mayores, mujeres y con menores ingresos familiares. La diabetes afecta negativamente la gravedad de la tuberculosis pulmonar, provocando síntomas más graves. Conclusión: la evidencia encontrada tiene el potencial de orientar las intervenciones de salud y permitir la implementación de acciones de salud más efectivas.

Descriptores: Epidemología; Tuberculosis; Diabetes Mellitus; Comorbilidad.

\section{INTRODUÇÃO}

O panorama epidemiológico da tuberculose (TB) apresenta-se impactante em termos de saúde pública, visto que dados da Organização Mundial da Saúde (OMS), em 2017, revelaram a existência de 10 milhões de pessoas com TB e 1,6 milhão de mortes, além de um terço da população estar infectada e apresentar $10 \%$ de chance de desenvolver a doença ao longo da vida ${ }^{1}$.

O maior risco de adoecimento pela TB está nos primeiros dois anos após a primoinfecção, mas o período de latência pode se estender até décadas e alguns fatores relacionados ao sistema imunológico podem aumentar o risco de adoecimento, dentre eles, destacam-se a infecção pelo vírus da imunodeficiência humana (HIV), doenças ou tratamentos imunossupressores, idade (menor do que 2 anos ou maior do que 60 anos) e o diabetes mellitus (DM) ${ }^{2}$.

Pesquisa financiada pelo Programa Nacional de Cooperação Acadêmica na Amazônia (PROCAD Amazônia)/Coordenação de Aperfeiçoamento de Pessoal de Nível Superior (CAPES) e da Fundação de Amparo à Pesquisa e ao Desenvolvimento Científico e Tecnológico do Maranhão (FAPEMA)

Autor correspondente: Giana Gislanne da Silva de Sousa. E-mail: giana.s@outlook.com.

Editora responsável: Mercedes Neto. 
O DM, destaca-se como uma doença metabólica de origem multifatorial, caracterizada pelos altos níveis de glicose no sangue, além disso fragiliza o sistema imunológico do indivíduo, deixando-o mais susceptível às infecções. E, assim como a TB, também é uma prioridade de Saúde Pública ${ }^{3}$, estando associado a várias complicações como o acidente vascular encefálico, a doença cardiovascular, a insuficiência renal e a ferida crônica ${ }^{4}$. Especificamente no ano de 2019 , estimou-se que em todo o mundo havia 463 milhões de pessoas com DM e, aproximadamente 4,2 milhões de mortes no mundo foram atribuídas a esta doença ${ }^{5}$

Verifica-se que os portadores de DM possuem cerca de três vezes mais chances de desenvolver TB ativa e, mesmo quando estes realizam o tratamento adequado, existe um aumento do risco de reinfecção ou óbito ${ }^{2,6}$. Acrescenta-se ainda que a distribuição espacial desta comorbidade no mundo pode ser explicada pelos fatores sociodemográficos e econômicos por existir uma forte relação entre as doenças e as condições sociais e determinantes de saúde ${ }^{7,8}$. Outrossim, as evidências atuais sugerem que, além dos fatores de risco individuais e sociais, fatores ecológicos, geográficos, climáticos e socioeconômicos incrementam a prevalência da comorbidade ${ }^{6,8}$.

Nessa perspectiva, as informações acerca de características sociodemográficas, clínicas e de indicadores epidemiológicos de morbidade (incidência e prevalência) dos casos associados de TB e DM permitem compreender a sua dinâmica no espaço e no espaço-tempo e as associações com as características locais ${ }^{9}$, apontando áreas e populações susceptíveis para sua ocorrência.

Ressalta-se que tais informações podem contribuir para que os profissionais e serviços de saúde promovam melhor controle da comorbidade, por servirem como um instrumento para auxiliar no gerenciamento e planejamento de estratégias de saúde direcionadas para o controle e prevenção de doenças ${ }^{10}$. Desse modo, evidencia-se a necessidade de atualizar e expandir o conhecimento, por meio da análise da literatura e a inclusão de estudos que evidenciem a associação TB/DM.

O objetivo do presente estudo foi sintetizar as evidências acerca de características sociodemográficas, indicadores epidemiológicos de morbidade e manifestações clínicas e dos casos associados de TB e DM.

\section{MÉTODO}

Trata-se de uma revisão integrativa, método que visa fornecer uma visão abrangente sobre determinado tema, com a finalidade de compreender melhor um fenômeno preocupante ${ }^{11}$. Para sua elaboração, foram seguidas as etapas: elaboração da questão de pesquisa, busca de dados na literatura, avaliação dos dados, análise de dados e apresentação dos resultados.

Para guiar a coleta de dados utilizou-se a estratégia PICo (População ou problema, Fenômeno de interesse e Contexto). O Problema (P) abrangeu a comorbidade TB/DM, o Interesse (I), características sociodemográficas, indicadores epidemiológicos de morbidade e manifestações clínicas e o Contexto (Co) referiu-se aos estudos realizados mundialmente ${ }^{12}$. Desse modo, estabeleceu-se a questão norteadora: “Quais são as evidências científicas disponíveis na literatura sobre características sociodemográficas, indicadores epidemiológicos de morbidade e manifestações clínicas da comorbidade TB/DM no mundo?".

A busca na literatura ocorreu no mês de agosto de de 2020 nas bases de dados Scopus, Literatura Latino Americana e do Caribe em Ciências da Saúde (LILACS) e Medical Literature Analysis and Retrieval System Online (MEDLINE) por meio do portal Biblioteca Virtual em Saúde Brasil (BVS) e no Scientific Eletronic Library Online (SciELO). Foram empregados os Descritores em Ciências da Saúde (DECS): "Tuberculose", "Diabetes Mellitus", "Epidemiologia" e "Sinais e Sintomas". Também foram cruzados seus correspondentes em inglês do Medical Subject Headings (MeSH) da National Library: Tuberculosis, Diabetes Mellitus", Epidemiology e "Signs and Symptoms". Os descritores foram combinados utilizando os operadores booleanos AND e OR. Os cruzamentos foram realizados da seguinte forma: "Tuberculosis" AND "Diabetes Mellitus") AND ("Epidemiology" OR “Signs and Symptoms").

Para seleção dos estudos foram adotados os seguintes critérios de inclusão: artigos científicos originais, disponibilizados de forma gratuita na íntegra, nos idiomas português, inglês ou espanhol, sem delimitação de tempo. Foram excluídos os editoriais, cartas ao editor, resumos, opinião de especialistas, revisões, resenhas, livros, capítulos de livros, teses e dissertações.

Os estudos foram pré-selecionados por meio de uma leitura minuciosa dos títulos e dos resumos para identificar os que apresentavam relação com a questão norteadora da revisão e com os critérios de inclusão e exclusão adotados. A estratégia de busca foi fundamentada pelo Preferred Reporting Items for systematic reviews and meta-analyses $(\text { PRISMA })^{13}$.

Para a análise e extração dos dados utilizou-se um instrumento validado que contempla os dados referentes a identificação do artigo, local de realização do estudo, características metodológicas e avaliação do rigor científico ${ }^{14}$. 
Na etapa de avaliação dos estudos, o rigor científico foi analisado considerando o delineamento de pesquisa de cada estudo para a identificação do nível de evidência, baseado no sistema de classificação de evidências que categoriza os estudos de forma hierárquica de acordo com a abordagem metodológica ${ }^{15}$. Tal escolha foi fundamentada por esse sistema proporcionar subsídios para avaliação crítica de estudos realizados para tomada de decisão no tocante a implementação das evidências científicas à prática clínica. Desse modo, os artigos foram classificados em: I- aqueles que apresentaram evidências oriundas de revisões sistemáticas ou meta-análise de relevantes ensaios clínicos; II- evidências derivadas de pelo menos um ensaio clínico randomizado controlado bem delineado; III- ensaios clínicos bem delineados sem randomização; IV- estudos de coorte e de caso-controle bem delineados; V- revisão sistemática de estudos descritivos e qualitativos; VIevidências derivadas de um único estudo descritivo ou qualitativo; VII- opinião de autoridades ou relatório de comitês de especialistas. Ressalta-se que as evidências de níveis I e II são consideradas fortes, níveis III a V moderadas e VI a VII fracas ${ }^{15}$.

\section{RESULTADOS}

A partir dos critérios estabelecidos para revisão integrativa, foram selecionados 16 estudos, publicados entre os anos de 2009 e 2020. Os resultados encontrados na busca estão expostos no fluxograma (Figura 1), adaptado do PRISMA ${ }^{13}$.

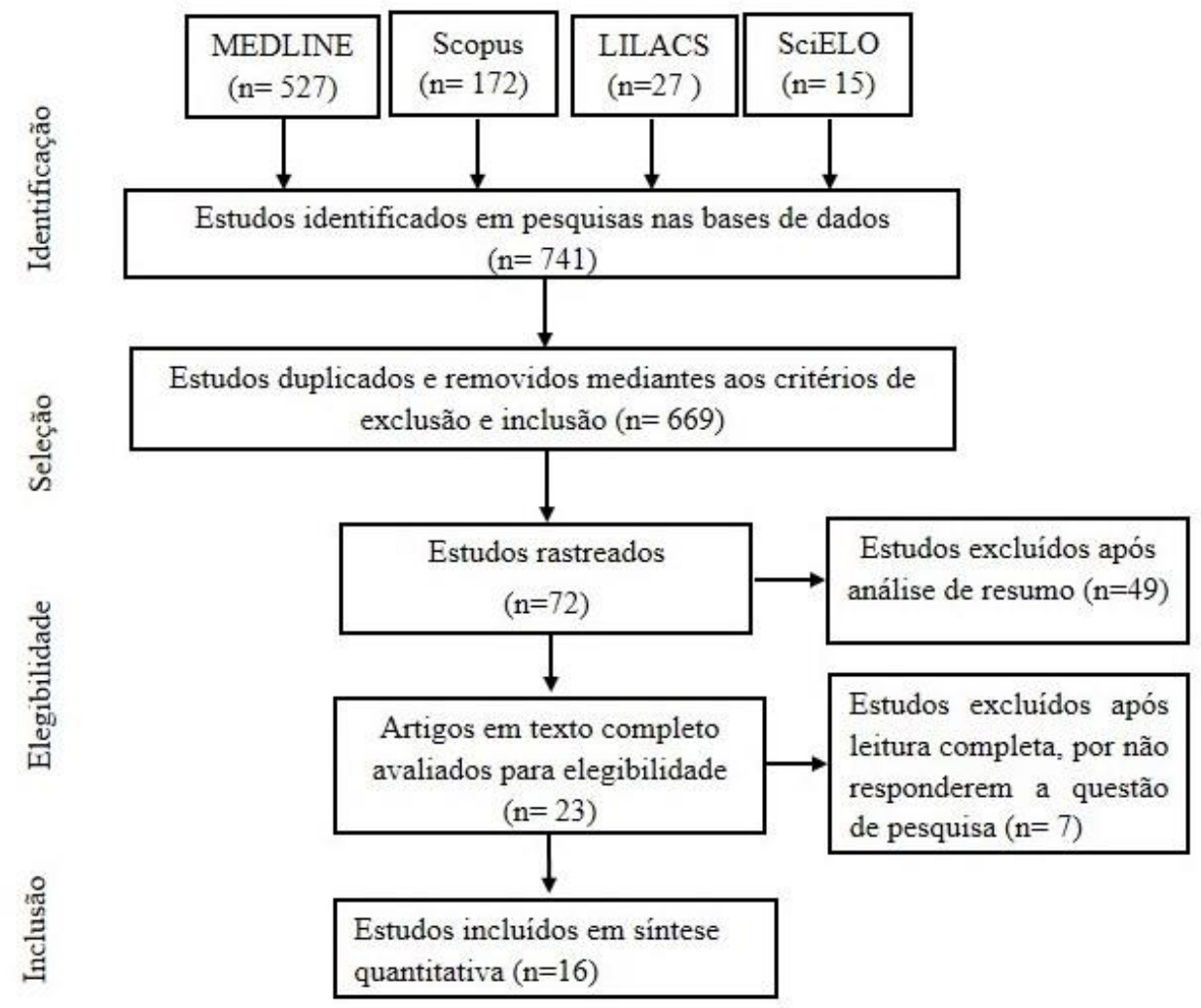

FIGURA 1: Fluxograma de seleção dos estudos encontrados. São Luís, MA, Brasil, 2020

A maioria absoluta (93,7\%) das pesquisas objetivou descrever e comparar as características clínicas e epidemiológicas dos pacientes com TB e DM, e apenas um artigo abordou a temática da análise espacial.

Sobre o cenário dos estudos, destaca-se que seis foram realizados no Brasil ${ }^{16-21}$, três no México ${ }^{22-24}$ e os outros na Índia ${ }^{24}$, Etiópia ${ }^{26}$, Espanha ${ }^{27}$, China $^{28}$, Chile ${ }^{29}$ e Turquia $^{30}$, apenas um analisou estimativas de diversos países ${ }^{31}$.

Quanto ao nível de evidência, sete $(43,7 \%)$ foram classificados como nível IV, sendo cinco de coorte retrospectiva e dois de coorte prospectiva, e 9 (56,25\%) foram classificados como nível VI, envolvendo a maioria dos estudos de natureza transversal, demostrando que os estudos elegíveis foram classificados com evidências moderadas e fracas.

Considerando os indicadores epidemiológicos de morbidade, os estudos revelaram que algumas regiões do mundo com alta prevalência de DM apresentaram maior incidência de TB ${ }^{18,20,21,26,27,29-31}$. A prevalência da TB entre os pacientes com DM foi maior do que na população em geral ${ }^{18,26,31}$ e um estudo apontou que os pacientes com DM apresentaram mais chance de ter $\mathrm{TB}^{18}$. 
Em se tratando de características sociodemográficas dos casos, a comorbidade esteve associada com a idade avançada $^{16-22,24-27,30}$, sexo feminino ${ }^{16-18,24-26,29}$ e renda familiar baixa ${ }^{18,25}$.

Em relação aos aspectos clínicos, pacientes comórbidos apresentaram maior gravidade nos sintomas do que aqueles sem DM, sendo mais frequentes: tosse, expectoração, hemoptise, febre, fadiga, sudorese noturna, fraqueza, diarreia, cefaleia, dores nas pernas e mal-estar ${ }^{18,19}$. Além disso, tiveram maior chance de apresentar padrões cavitários no raio $X$ de tórax, baciloscopia positiva ${ }^{17,19,21,22,27-28,30}$, atraso na conversão do escarro ${ }^{19-22}$, falha no tratamento ${ }^{16,24,26}$ e maior chance de desenvolver tuberculose multirresistente $(T B M R)^{16}$.

Evidenciou-se ainda que existe agregação espacial dos pacientes com DM/TB em torno do centro de saúde onde receberam cuidados $^{23}$. As Figuras 2 e 3 destacam os autores, países de realização dos estudos, níveis de evidência, assim como os objetivos e os principais achados nos estudos selecionados.

\begin{tabular}{|c|c|c|c|c|}
\hline $\begin{array}{c}\text { Autores / } \\
\text { Local }\end{array}$ & $\begin{array}{l}\text { Nível de } \\
\text { evidência }\end{array}$ & $\begin{array}{l}\text { População (N) e/ou } \\
\text { Amostra (n) }\end{array}$ & Objetivos & Principais Achados \\
\hline $\begin{array}{l}\text { Evangelista } \\
\text { et al, Brasil, } \\
2020^{16}\end{array}$ & IV & $\begin{array}{l}\text { Casos notificados } \\
\text { ao Sistema de } \\
\text { Informação de } \\
\text { Agravos de } \\
\text { Notificações - } \\
\text { SINAN (N= } \\
\text { 709.429). }\end{array}$ & $\begin{array}{l}\text { Conhecer a situação } \\
\text { clínico-epidemiológica } \\
\text { dos casos notificados de } \\
\text { TB/DM por faixa etária no } \\
\text { Brasil. }\end{array}$ & $\begin{array}{l}\text { A comorbidade TB/DM foi encontrada em } \\
6,0 \% \text { dos casos, principalmente em homens } \\
\text { de } 18 \text { a } 59 \text { anos, entretanto as mulheres } \\
\text { adolescentes e as mulheres mais velhas } \\
\text { apresentaram uma chance maior de ter } \\
\text { TB/DM. Observou-se um aumento da TBMR } \\
\text { em todas as faixas etárias entre os pacientes } \\
\text { com TBDM. }\end{array}$ \\
\hline $\begin{array}{l}\text { Abreu et al, } \\
\text { Brasil } \\
2020^{17}\end{array}$ & IV & $\begin{array}{l}\text { Casos de } \\
\text { tuberculose e } \\
\text { diabetes notificados } \\
\text { nas bases nacionais } \\
\text { do Sinan e do } \\
\text { Hiperdia ( } \mathrm{N}= \\
338.825 \text { ) }\end{array}$ & $\begin{array}{l}\text { Analisar o perfil } \\
\text { sociodemográfico e as } \\
\text { características do } \\
\text { diagnóstico e tratamento } \\
\text { dos casos de tuberculose } \\
\text { com e sem diabetes no } \\
\text { Brasil. }\end{array}$ & $\begin{array}{l}\text { A comorbidade foi encontrada em } 7,2 \% \text { dos } \\
\text { casos. Houve associação com o sexo } \\
\text { feminino, nas faixas etárias } 40-59 \text { anos e } \geq 60 \\
\text { e resultado positivo da baciloscopia. }\end{array}$ \\
\hline $\begin{array}{l}\text { Leal et al, } \\
\text { Brasil, } \\
2017^{18}\end{array}$ & $\mathrm{VI}$ & $\begin{array}{l}\text { Indivíduos em } \\
\text { tratamento para } \\
\text { tuberculose em } \\
\text { Salvador }(n=323)\end{array}$ & $\begin{array}{l}\text { Descrever e comparar as } \\
\text { características clínicas e } \\
\text { epidemiológicas dos } \\
\text { pacientes com TB, com e } \\
\text { sem diabetes. }\end{array}$ & $\begin{array}{l}\text { A prevalência da comorbidade foi de } 14,6 \% \text {, } \\
\text { este grupo era mais velho tinham um nível } \\
\text { educacional mais elevado e uma renda } \\
\text { familiar mais baixa. Os pacientes } \\
\text { apresentaram cicatrização lenta de feridas, } \\
\text { fadiga, dores no corpo e maior média de } \\
\text { duração da febre e outros sintomas. }\end{array}$ \\
\hline $\begin{array}{l}\text { Gil-Santana } \\
\text { et al, Brasil. } \\
2016^{19}\end{array}$ & IV & $\begin{array}{l}\text { Casos de TB } \\
\text { atendidos no } \\
\text { IBT }(\mathrm{N}=408)\end{array}$ & $\begin{array}{l}\text { Comparar a apresentação } \\
\text { clínica e os desfechos da } \\
\text { TB em pacientes } \\
\text { diabéticos e não } \\
\text { diabéticos atendidos em } \\
\text { centros de referência de } \\
\text { atendimento primário em } \\
\text { uma área altamente } \\
\text { endêmica de TB no Brasil. }\end{array}$ & $\begin{array}{l}\text { Os pacientes com DM eram mais velhos, } \\
\text { apresentaram maior frequência de tosse, } \\
\text { sudorese noturna, hemoptise e mal-estar e } \\
\text { maior frequência de baciloscopia positiva. A } \\
\text { maior gravidade da TB foi associados à } \\
\text { comorbidade TB-diabetes. }\end{array}$ \\
\hline $\begin{array}{l}\text { Reis-Santos } \\
\text { et al, Brasil, } \\
2014^{20}\end{array}$ & VI & $\begin{array}{l}\text { Casos de TB } \\
\text { reportados ao } \\
\text { SINAN (N= } \\
990.017)\end{array}$ & $\begin{array}{l}\text { Avaliar os fatores } \\
\text { sociodemográficos e } \\
\text { clínicos que podem } \\
\text { influenciar o resultado da } \\
\text { TB em pacientes com DM. }\end{array}$ & $\begin{array}{l}\text { A chance de morte foi maior para os } \\
\text { indivíduos com } 60 \text { anos de idade, } \\
\text { institucionalizado em abrigo, alcoolistas, } \\
\text { HIV/AIDS, TB pulmonar e extrapulmonar, com } \\
\text { tipo de tratamento desconhecido. }\end{array}$ \\
\hline $\begin{array}{l}\text { Reis-Santos } \\
\text { et al, Brasil, } \\
2013^{21}\end{array}$ & VI & $\begin{array}{l}\text { Casos de TB } \\
\text { reportados ao } \\
\text { SINAN }(N=84.691)\end{array}$ & $\begin{array}{l}\text { Avaliar as diferenças } \\
\text { sociodemográficas e } \\
\text { clínicas em pacientes com } \\
\text { TB com e sem DM. }\end{array}$ & $\begin{array}{l}\text { Os sujeitos com TB/DM eram mais velhos, } \\
\text { apresentavam teste de baciloscopia positiva } \\
\text { inicial e maior probabilidade de morrer de TB, } \\
\text { tinham menos probabilidade de ter sido } \\
\text { institucionalizados, maior chance de } \\
\text { desenvolver TB extra pulmonar e retornar ao } \\
\text { tratamento de TB após abandono. }\end{array}$ \\
\hline
\end{tabular}

IBIT=Instituto Brasileiro para Investigação da Tuberculose em Salvador; SINAN=Sistema de Informação de Agravos de Notificação; TBMR= tuberculose multirresistente. FIGURA 2: Distribuição dos artigos nacionais incluídos na revisão. São Luís, MA, Brasil, 2020 


\begin{tabular}{|c|c|c|c|c|}
\hline $\begin{array}{c}\text { Autores / } \\
\text { Local }\end{array}$ & $\begin{array}{l}\text { Nível de } \\
\text { evidência }\end{array}$ & $\begin{array}{l}\text { População (N) e/ou } \\
\text { Amostra (n) }\end{array}$ & Objetivos & Principais Achados \\
\hline $\begin{array}{l}\text { Jiménez- } \\
\text { Corona et } \\
\text { al, México, } \\
2012^{22}\end{array}$ & IV & $\begin{array}{l}\text { Indivíduos com } \\
\text { tuberculose no sul do } \\
\text { México ( } n=1262)\end{array}$ & $\begin{array}{l}\text { Determinar as consequências } \\
\text { clínicas da tuberculose } \\
\text { pulmonar (TBP) entre } \\
\text { pacientes com (DM). }\end{array}$ & $\begin{array}{l}\text { A prevalência de } \mathrm{DM} \text { foi de } 29,63 \% \text {. Pacientes } \\
\text { comórbidos apresentaram manifestações clínicas } \\
\text { mais graves como: cavidades de qualquer } \\
\text { tamanho na radiografia de tórax, retardo na } \\
\text { conversão de escarro, maior probabilidade de } \\
\text { falha no tratamento recorrência e recidiva. }\end{array}$ \\
\hline $\begin{array}{l}\text { Blanco- } \\
\text { Guillot et } \\
\text { al, México, } \\
2018^{23}\end{array}$ & IV & $\begin{array}{l}\text { Indivíduos com TB } \\
\text { pulmonar registrados } \\
\text { no Registro Nacional } \\
\text { de Tuberculose } \\
\text { ( } \mathrm{n}=1105) .\end{array}$ & $\begin{array}{l}\text { Testar a hipótese de que a } \\
\text { DM e TBP ocorrem em } \\
\text { agregações espaciais e } \\
\text { moleculares. }\end{array}$ & $\begin{array}{l}\text { Identificou-se agrupamento molecular e } \\
\text { agregação espacial dos pacientes com DM e TB. }\end{array}$ \\
\hline $\begin{array}{l}\text { Delgado- } \\
\text { Sánchez et } \\
\text { al, México, } \\
2015^{24}\end{array}$ & VI & $\begin{array}{l}\text { Casos de TB pulmonar } \\
\text { contidos no Registro } \\
\text { Nacional de } \\
\text { Tuberculose } \\
(\mathrm{N}=181.378)\end{array}$ & $\begin{array}{l}\text { Descrever as condições } \\
\text { clínicas de TB pulmonar } \\
\text { associadas e não associadas à } \\
\text { DM e a compilação dos } \\
\text { resultados do tratamento em } \\
\text { pacientes com e sem DM. }\end{array}$ & $\begin{array}{l}\text { As incidências de TB pulmonar associadas ao DM } \\
\text { aumentaram, contrastando com as taxas de TB } \\
\text { pulmonar sem DM, que diminuiram. Os } \\
\text { pacientes com TB/DM eram mais propensos a } \\
\text { serem mulheres, idosos, residentes na região } \\
\text { urbana e apresentaram maior probabilidade de } \\
\text { falha terapêutica. }\end{array}$ \\
\hline $\begin{array}{l}\text { Pande et } \\
\text { al, Índia, } \\
2019^{25}\end{array}$ & IV & $\begin{array}{l}\text { Adultos hospitalizados } \\
\text { com diagnóstico de TB } \\
\text { pulmonar (PTB) ou TB } \\
\text { extrapulmonar }\end{array}$ & $\begin{array}{l}\text { Estimar a prevalência de DM } \\
\text { em pacientes adultos com TB } \\
\text { hospitalizados e avaliar os } \\
\text { fatores associados a maior } \\
\text { prevalência de DM entre } \\
\text { pacientes com TB. }\end{array}$ & $\begin{array}{l}\text { A prevalência geral de DM entre os pacientes } \\
\text { com TB foi de } 25,3 \% \text {. Pacientes com TB com } \\
\text { idade acima de } 41 \text { anos tiveram maior chance de } \\
\text { apresentar DM. As mulheres tiveram menor } \\
\text { chance de apresentar DM do que os pacientes do } \\
\text { sexo masculino com TB. }\end{array}$ \\
\hline $\begin{array}{l}\text { Workneh } \\
\text { et al, } \\
\text { Etiópia } \\
2016^{26}\end{array}$ & VI & $\begin{array}{l}\text { Pacientes com TB } \\
\text { recém-diagnosticados } \\
\text { que frequentavam } \\
\text { clínica de tratamento } \\
\text { ( } n=1314 \text { ). }\end{array}$ & $\begin{array}{l}\text { Determinar a prevalência e } \\
\text { analisar os fatores associados } \\
\text { à comorbidade de TB e DM } \\
\text { na região de Amhara do } \\
\text { Sudeste, Etiópia. }\end{array}$ & $\begin{array}{l}\text { A prevalência de DM foi estimada em } 8,3 \% \text { entre } \\
\text { o paciente com TB. Os fatores associados a } \\
\text { comorbidade TB-DM foram sexo feminino, idade } \\
\text { maior que } 41 \text { anos, TB pulmonar e com história } \\
\text { familiar de DM. }\end{array}$ \\
\hline $\begin{array}{l}\text { Moreno- } \\
\text { Martínez } \\
\text { el al, } \\
\text { Espanha, } \\
2015^{27}\end{array}$ & $\mathrm{VI}$ & $\begin{array}{l}\text { Casos diagnosticados } \\
\text { com TB ativa } \\
\text { detectados pelo } \\
\text { Programa para } \\
\text { Prevenção e Controle } \\
\text { da Tuberculose } \\
\text { (N=5849). }\end{array}$ & $\begin{array}{l}\text { Analisar a prevalência de DM } \\
\text { e seus fatores associados em } \\
\text { adultos com TB em uma } \\
\text { cidade grande de um país } \\
\text { industrializado. }\end{array}$ & $\begin{array}{l}\text { A prevalência variou de } 4,0 \% \text { a } 7,2 \% \text {. Os fatores } \\
\text { associados a TB/DM foram: nascidos na Espanha, } \\
\text { idade maior que } 40 \text { anos e padrões cavitários no } \\
\text { raio } X \\
\text { de tórax. }\end{array}$ \\
\hline $\begin{array}{l}\text { Hongguan } \\
\text { et al, } \\
\text { China, } \\
2014^{28}\end{array}$ & IV & $\begin{array}{l}\text { Indivíduos com TB } \\
\text { pulmonar registrados } \\
\text { no programa de } \\
\text { controle para TB em } \\
\text { Pequim ( } n=1126) \text {. }\end{array}$ & $\begin{array}{l}\text { Explorar o impacto do DM na } \\
\text { apresentação clínica e no } \\
\text { resultado do tratamento da } \\
\text { tuberculose pulmonar (TBP) } \\
\text { na China. }\end{array}$ & $\begin{array}{l}\text { O DM estava associado a maior proporção de } \\
\text { baciloscopia positiva, maior proporção de } \\
\text { cavidades pulmonares e aos sintomas: tosse, } \\
\text { expectoração, hemoptise, sudorese noturna e } \\
\text { fraqueza. }\end{array}$ \\
\hline $\begin{array}{l}\text { Herrera et } \\
\text { al, Chile, } \\
2013^{29}\end{array}$ & VI & $\begin{array}{l}\text { Casos de tuberculose } \\
\text { notificados no Registro } \\
\text { Nacional de } \\
\text { Tuberculose de uma } \\
\text { região metropolitana } \\
(\mathrm{N}=821) \text {. }\end{array}$ & $\begin{array}{l}\text { Determinar a prevalência de } \\
\text { DM entre os casos de TB } \\
\text { diagnosticados em } 2012 \text { em } \\
\text { Santiago do Chile na área } \\
\text { metropolitana, e estimar a } \\
\text { associação dessas duas } \\
\text { doenças. }\end{array}$ & $\begin{array}{l}\text { A prevalência de DM foi de } 15,6 \% \text {. A taxa de } \\
\text { incidência estimada de TB entre a população } \\
\text { diabética é } 1,7 \text { vezes maior que a população } \\
\text { geral da região. }\end{array}$ \\
\hline $\begin{array}{l}\text { Tatar et al, } \\
\text { Turquia, } \\
2009^{30}\end{array}$ & VI & $\begin{array}{l}\text { Casos de TB } \\
\text { registrados no } \\
\text { Dispensário de } \\
\text { Tuberculose Esrefpasa } \\
\text { em Izmir, Turquia } \\
(\mathrm{N}=1.063) \text {. }\end{array}$ & $\begin{array}{l}\text { Avaliar as características da } \\
\text { TB em diabéticos na região. }\end{array}$ & $\begin{array}{l}\text { A prevalência foi de } 7,3 \% \text { de TB em pacientes } \\
\text { com DM. A formação de cavidades e a } \\
\text { localização atípica foram mais encontradas em } \\
\text { diabéticos, além de maior duração do } \\
\text { tratamento e taxa de resistência a drogas. }\end{array}$ \\
\hline $\begin{array}{l}\text { Badawi et } \\
\text { al, Mundo, } \\
2014^{31}\end{array}$ & VI & $\begin{array}{l}\text { Estimativas de } \\
\text { incidência e } \\
\text { prevalência da TB e } \\
\text { DM em } 196 \text { países. }\end{array}$ & $\begin{array}{l}\text { Examinar a relação global } \\
\text { entre a prevalência de DM e } \\
\text { a incidência de TB para } \\
\text { avaliar sua coexistência em } \\
\text { todo o mundo. }\end{array}$ & $\begin{array}{l}\text { Os países com alta prevalência de DM } \\
\text { apresentaram associação significativa com a } \\
\text { incidência de TB. }\end{array}$ \\
\hline
\end{tabular}

FIGURA 3: Distribuição dos artigos internacionais incluídos na revisão. São Luís, MA, Brasil, 2020 


\section{DISCUSSÃo}

Os estudos selecionados nesta revisão indicaram que existe associação positiva entre a prevalência de DM e elevados índices de TB ${ }^{18,20-23,26,27,29,31}$. Corroborando com tais achados, pacientes com DM tem maior risco de obter TB, com evidências consistentes de um aumento de duas a quatro vezes no risco de desenvolver TB ${ }^{32}$.

Foi evidenciado que o DM gera impacto nas taxas de TB em alguns países, sobretudo naqueles com alta prevalência de $\mathrm{DM}$, maior que $7,6 \%{ }^{31}$, como México $(33 \%)^{23}$, Índia $(24,3 \%)^{25}$ e Brasil $(14,6 \%)^{18}$. Achado este constatado em outros estudos, nos quais denotaram que países em desenvolvimento ou subdesenvolvidos, apresentaram prevalência elevada da comorbidade, a exemplo dos estudos realizados na Nigéria $(9,4 \%)^{8}$ e Peru $(11,1 \%)^{33}$.

Nesta revisão, a maioria dos estudos apontaram que a idade avançada estava associada à comorbidade TB/DM ${ }^{16-}$ 22,24-27,29, possivelmente deve-se ao fato de que ocorre um declínio no estado imunológico e alterações fisiológicas com avanço da idade, o que favorece tanto os agravos por doenças crônicas não transmissíveis, como por doenças infecciosas $^{34}$. Alterações da função pulmonar e no clearance mucociliar em pessoas de idade avançada podem favorecer uma reativação do bacilo de Koch que associada à imunossupressão causada pelo DM contribui no desenvolvimento da lesão tuberculosa ${ }^{35}$.

Além disso, a incidência da TB na faixa etária acima de 40 anos coincide com o adoecimento do DM, o que torna a idade um fator de risco para os pacientes com as ambas doenças, visto que a associação entre TB/DM proporciona a ocorrência de sinais clínicos mais graves em paciente com $\mathrm{TB}^{18,35}$. E este risco atinge principalmente o sexo feminino, visto que as mulheres acima de 60 anos têm $41 \%$ mais chance de ter DM do que mulheres em outras faixa etária ${ }^{16}$.

Relacionado a isto, evidenciou-se associação entre a ocorrência da comorbidade e o sexo feminino ${ }^{16,24}$ e apresentaram 1,7 vezes mais chances de ter a comorbidade quando comparadas ao sexo masculino ${ }^{26}$. Apontou-se que a prevalência de $19,1 \%$ entre as mulheres com diabetes foi maior que na população em geral ${ }^{29}$.

O sexo feminino apresenta uma maior prevalência de DM, possivelmente relacionada às questões de gênero, pois os homens apresentam um maior desconhecimento sobre o diagnóstico da doença e comumente frequentam menos os serviços de saúde, diferentemente da mulher, que gerencia a assistência à saúde da família, e acessa periodicamente os serviços de saúde ${ }^{36}$.

Ademais, aponta-se que a chance de o diabetes ser fator de risco entre as mulheres com tuberculose é maior do que para os homens ${ }^{29}$. Isto pode estar relacionado à má utilização dos serviços de saúde, ao cuidado das mulheres com os doentes e à influência do estrogênio na inibição de citocinas que provoca um efeito imunossupressor potencializado pelo DM, tais fatores aumentam a vulnerabilidade das mulheres à DM e, consequentemente, ao $\mathrm{TB}^{26}$.

Os estudos elegíveis revelaram forte relação da TB com as condições socioeconômicas desfavoráveis, visto que a concentração dos casos em determinadas regiões está relacionada com condições precárias de moradia, como casas mal ventiladas e aglomerados urbanos, fatores que determinam a influência da pobreza na transmissão do Mycobacterium tuberculosis ${ }^{9,18}$

Colaborando com tais dados, evidenciou-se que os pacientes com DM em países de renda média ou baixa no continente asiático em cenário de elevada taxa de incidência para TB, apresentaram maior risco de TB do que pacientes com DM em países de alta renda, como na região da Europa e nos Estados Unidos e Canadá 32 .

Sobre os aspectos clínicos, pacientes com a comorbidade apresentaram sinais e sintomas mais graves tendo em vista que o DM é um dos fatores de risco para o desenvolvimento de infecções, devido ao declínio na resposta imune, pois afeta as células de defesa do organismo, as células fagocíticas (macrófagos e linfócitos). Além disso, os níveis baixos de insulina e a hiperglicemia contribuem negativamente no controle dos bacilos da TB, pois alteram a função quimiotáxica, a fagocitose e a apresentação de antígenos ${ }^{3,37}$.

Ressalta-se que a frequência da febre foi três vezes maior entre os pacientes com TB e DM, este fato decorre da elevada carga bacteriana e tempo mais lento na negativação da baciloscopia após o início do tratamento ${ }^{19}$.

Sobre a ocorrência de cavidades pulmonares e positividade na baciloscopia, estudos sugerem que uma maior taxa de positividade ao esfregaço esteja associada a maior formação de cavidades e maior dano pulmonar devido ao $\mathrm{DM}^{19,29}$. Ademais, a recuperação das lesões pulmonares ocorre de maneira mais lenta no grupo de pacientes diabéticos quando comparado aos não diabéticos ${ }^{18}$.

Nessa perspectiva, aponta-se que o exame radiológico pode apresentar alterações consideradas atípicas e que as cavidades pulmonares são mais frequentes em paciente com a comorbidade ${ }^{38}$, pois estes apresentam uma maior carga bacilar e maior tempo na conversão da cultura, o que aumenta o risco de recidivas e óbito nesses pacientes ${ }^{19}$. 
Em relação à falha no tratamento, os achados desta revisão são congruentes com um estudo que investigou a resistência ao tratamento da TB e comprovou que os pacientes diabéticos desenvolveram com maior frequência resistência às drogas no tratamento da $\mathrm{TB}^{39}$.

É válido mencionar que estudos ecológicos contribuem para a análise de riscos socioambientais e identificação de áreas mais vulneráveis à ocorrência da comorbidade ${ }^{8}$. Alguns estudos evidenciaram a distribuição espacial da $\mathrm{TB}^{9,10} \mathrm{e}$ do $\mathrm{DM}^{40}$ isoladamente, porém ao considerar a infeç̧ão por TB em associação com o DM, a literatura apresenta-se escassa sobre a temática.

Estudo realizado em uma região do México de maior incidência de TB e alta prevalência de DM, utilizando a análise de Moran global, apontou uma distribuição não aleatória entre os pacientes com TB e DM que foram geoespacialmente agregados em torno do centro de saúde onde receberam cuidados de saúde ${ }^{23}$.

Ressalta-se que as tecnologias de georreferenciamento permitem a analise espacial e espaço-temporal dos casos de TB/DM e fornecem relevante contribuição para a saúde pública, pois os mapas obtidos revelam condições socioeconômicas relacionando-as com o processo saúde-doença no território ${ }^{9}$. Dessa forma, sugere-se que sejam realizadas investigações científicas com essa abordagem para que haja uma melhor compreensão sobre a distribuição desta comorbidade no território, considerando-se as desigualdades socioespaciais existentes, com vistas ao direcionamento de ações estratégicas de intervenções em saúde.

Cabe ressaltar que os estudos sobre a distribuição e características sociodemográficas de doenças são relevantes, pois servem como ferramentas que podem orientar as ações de vigilância em saúde, auxiliando na formulação de políticas públicas ${ }^{10}$. Neste sentido, a síntese de informações contidas na literatura acerca das características clínicoepidemiológicas da comorbidade TB/DM realizada por meio desta revisão integrativa, contribui para prática baseada em evidência por favorecer a ligação teoria e prática ${ }^{11}$, destacando pontos importantes a serem repensados $\mathrm{e}$ direcionados ao manejo clínico, controle e vigilância da comorbidade.

Destaca-se ainda que a maioria dos estudos selecionados foram classificados apresentando níveis de evidências moderados e fracos. É importante frisar que as mudanças e o embasamento da prática assistencial são pautados na produção de conhecimento científico, portanto, estudos com os melhores níveis de evidências são indispensáveis e essenciais para os serviços de saúde ${ }^{11-15}$. Desse modo, sugere-se que estudos com evidências fortes, a exemplo de ensaios clínicos randomizados, sejam desenvolvidos, sobretudo nas regiões endêmicas para TB que apresentam elevados índices de prevalência de DM.

\section{Limitações do estudo}

Como limitações dessa revisão integrativa cita-se a possibilidade de subnotificação da casos da comorbidade, pois a maioria dos estudos utilizaram dados secundários, e no caso dos estudos realizados no Brasil, o campo de agravo associado à TB nas fichas de notificação não é de preenchimento obrigatório, o que inviabiliza o conhecimento fidedigno da situação epidemiológica da comorbidade. Ressalta-se ainda a dificuldade de acesso a algumas publicações restritas, sendo incluído no estudo apenas textos de acesso livre, disponibilizados na íntegra. Além disso, observou-se a escassez de artigos que abordam a distribuição espacial e espaço-temporal dos casos da comorbidade TB/DM e a ausência de estudos com níveis de evidências fortes.

\section{CONCLUSÃO}

Os achados apontaram que o DM afeta negativamente o controle da TB, pois contribui para aumento nas taxas de infecção pelo Mycobacterium tuberculosis em algumas regiões do mundo, sobretudo em países em desenvolvimento, provocando sintomas mais graves da TB e dificultando seu tratamento. Além disso, esta comorbidade encontra-se distribuída heterogeneamente no espaço, atingindo grupos de maior vulnerabilidade social.

Ressalta-se que a implementação de medidas com objetivo de controlar a crescente epidemia de DM, com enfoque nos principais fatores de risco, pode influenciar de forma benéfica nos índices de TB, sobretudo nas regiões onde existe forte associação entre tais afecções. Tais evidências apontadas têm potencial para guiar as intervenções em saúde e possibilitam a implementação de ações de saúde mais efetivas.

\section{REFERÊNCIAS}

1. World Health Organization (WHO). Guidelines on tuberculosis infection prevention and control, 2019 update. Geneva: WHO [internet], 2019 [cited 2019 Dec 10]. Avalaible from:

https://apps.who.int/iris/bitstream/handle/10665/311259/9789241550512-eng.pdf?ua=1\&ua=1

2. Silva DR, Muñoz-Torrico M, Duarte R, Galvão T, Bonini EH, Arbex FF, Arbex MA, Augusto VMa, Rabahi MF, Melo FCQ. Risk factors for tuberculosis: diabetes, smoking, alcohol use, and the use of other drugs. J. bras. pneumol. [Internet], 2018 [cited 2019 Dec 10]; 44 (2): 145-52. DOI: http://dx.doi.org/10.1590/s1806-37562017000000443 
3. Kumar NP, Sridhar R, Nair D, Banurekha VV, Nutman TB, Babu S. Type 2 diabetes mellitus is associated with altered CD8+ T and natural killer cell function in pulmonary tuberculosis. Immunology [Internet], 2015 [cited 2019 Dec 10]; 144 (4): 677-86. DOI: https://doi.org/10.1111/imm.12421

4. Tonetto IFA, Batista MHB, Gomides DS, Pace AE. Quality of life of people with diabetes mellitus. Rev. esc. enferm. USP [Internet], 2019 [cited 2019 Dec 10]; (53): 1-8. DOI: http://dx.doi.org/10.1590/s1980-220x2018002803424

5. International Diabetes Federation. IDF Diabetes Atlas. $9^{\text {th }}$ ed. Brussels, Belgium: International Diabetes Federation [Internet], 2019 [cited 2019 Dec 10]. Avalaible from: https://diabetesatlas.org/en/

6. Pereira SM, Araújo GS, Souza CA, Santos T, Oliveira MG, Barreto ML. Association between diabetes and tuberculosis: casecontrol study. Rev. Saúde Pública [Internet], 2016 [cited 2019 Dec 10]; 50 (82):1-7. DOI: https://doi.org/10.1590/S15188787.2016050006374

7. Ekeke KN, Ukwaja K, Chukwu JN, Nwafor CC, Meka AO, Egbagbe EE. Screening for diabetes mellitus among tuberculosis patients in Southern Nigeria: a multi-centre implementation study under programme settings. Sci Rep [Internet], 2017 [cited 2019 Dec 10]; (7): 1-8. DOI: https://doi.org/10.1038/srep44205

8. Ibiapina É, Bernardes, A. Health maps and the contemporary visibility regime. Saude soc. [Internet], 2019 [cited 2019 Dec 10]; 28 (1): 322-36. DOI: https://doi.org/10.1590/S0104-12902019170982

9. Pereira AGL, Escosteguy CC, Valencia LIO, Magalhães MAFM, Medronho RA. Spatial analysis of tuberculosis cases and association with socioeconomic factors: an experience in the city of Rio de Janeiro. Cad. saúde colet. [Internet], 2018 [cited 2019 Dec 10]; 26 (2): 203-10. DOI: http://dx.doi.org/10.1590/1414-462x201800020013

10. Leal BN, Rodrigues ILA, Nogueira LMV, Mesquita CR, Caldas RJC, Santos BO. Controle da tuberculose, serviços de atenção primária em saúde e o geoprocessamento: revisão integrativa da literatura. Rev. G\&S [Internet], 2019 [cited 2020 Jul 14]; 10 (1): 22-32, 2019. DOI: https://doi.org/10.26512/gs.v10i1.22563

11. Ercole FF, Melo LSD, Alcoforado CLGC. Integrative review versus systematic review. Rev. Min. Enferm. [Internet], 2014 [cited 2019 Dec 10]; 18(1): 9-11. DOI: http://www.dx.doi.org/10.5935/1415-2762.20140001

12. The Joanna Briggs Institute (JBI). The Joanna Briggs Institute Reviewers' Manual 2015: methodology for JBI scoping reviews. Adelaide: JBI; 2015. [cited 2019 Dec 10]. Available from: https://nursing.Isuhsc.edu/JBI/docs/ReviewersManuals/Scoping-.pdf

13. Galvão TF, Pansani TSA, Harrad D. Principais itens para relatar Revisões sistemáticas e Meta-análises: A recomendação PRISMA. Epidemiol. Serv. Saúde [Internet], 2015 [cited 2019 Dec 10]; 24 (2): 335-42. DOI: http://dx.doi.org/10.5123/S167949742015000200017

14. Silveira, RCCP. O cuidado de enfermagem e o cateter de Hickman: a busca de evidências [Master thesis]. Ribeirão Preto (SP): Universidade de São Paulo; 2005.

15. Melnyk BM, Fineout-overholt E. Making. The case for evidence-based practice. In: Melnyk BM, Fineout-Overholt E. Evidencebased practice in nursing \& healthcare: a guide to best practice. Philadelphia: Lippincot Williams \& Wilkins; 2011.

16. Evangelista MSN, Maia R, Toledo JP, Abreu RG, Barreira D. Tuberculosis associated with diabetes mellitus by age group in Brazil: a retrospective cohort study, 2007-2014. Braz. J. Infect. Dis. [Internet], 2020 [cited 2020 Aug 13]; 24(2): 130-6. DOI: https://doi.org/10.1016/j.bjid.2020.03.005

17. Abreu RG, Rolim LS, Sousa AA, Oliveira MRF. Tuberculosis and diabetes: association with sociodemographic characteristics and diagnosis and treatment of tuberculosis. Brazil, 2007-2011. Rev. bras. epidemiol. [Internet], 2020 [cited 2020 Aug 13]; 23. DOI: http://dx.doi.org/10.1590/1980-549720200009

18. Leal SB, Araújo GS, Nery JS, Santos CAT, Oliveira MG, Barreto ML, Pereira SM. Clinical and epidemiological aspects of cases of tuberculosis associated with diabetes in Salvador, Bahia, Brazil. Rev. Soc. Bras. Med. Trop. [Internet], 2017 [cited 2019 Dec 10]; 50 (3): 408-12. DOI: http://dx.doi.org/10.1590/0037-8682-0409-2016

19. Gil-Santana L, Almeida-Junior JL, Oliveira CAM, Hickson LS, Daltro C, Castro S, Kornfeld HE, et al. Diabetes Is Associated with Worse Clinical Presentation in Tuberculosis Patients from Brazil: A Retrospective Cohort Study. PLoS ONE [Internet], 2016 [cited 2019 dez 4]; 11 (1): 1-13. DOI: https://doi.org/10.1371/journal.pone.0146876

20. Reis-Santos B, Gomes T, Locatelli R, Oliveira ER, Sanchez MN, Horta BL, et al. Treatment Outcomes in Tuberculosis Patients with Diabetes: A Polytomous Analysis Using Brazilian Surveillance System. PloS One [Internet], 2014 [cited 2019 Dec 10]; 9 (7): 1-9. DOI: https://doi.org/10.1371/journal.pone.0100082

21. Reis-Santos B, Locatelli R, Horta BL, Faerstein E, Sanchez MN, Riley LW, Maciel EL. Socio-Demographic and Clinical Differences in Subjects with Tuberculosis with and without Diabetes Mellitus in Brazil - A Multivariate Analysis. PloS One [Internet], 2013 [cited 2019 dez 04]; 8(4): 1-6. DOI: https://doi.org/10.1371/journal.pone.0062604.

22. Jiménez-Corona ME, Cruz-Hervert LP, García-García L, Ferreyra-Reyes L, Delgado-Sánchez G, Bobadilla-del-Valle M, et al. Association of diabetes and tuberculosis: impact on treatment and post-treatment outcomes. Thorax [Internet], 2013 [cited 2019 Dec 10]; 68 (3): 214-20. DOI: http://dx.doi.org/10.1136/thoraxjnl-2012-201756

23. Blanco-Guillot F, Castañeda-Cediel ML, Cruz-Hervert P, Ferreyra-Reyes L, Delgado-Sánchez G, Ferreira-Guerrero E, et al. Genotyping and spatial analysis of pulmonary tuberculosis and diabetes cases in the state of Veracruz, Mexico. PloS One [Internet], 2018 [cited 2019 Dec 10]; 13(3): 1-20. DOI: https://doi.org/10.1371/journal.pone.0193911

24. Delgado-Sánchez G, García-García L, Castellanos-Joya M, Cruz-Hervert P, Ferreyra-Reyes L, Ferreira-Guerrero E, et al. Association of Pulmonary Tuberculosis and Diabetes in Mexico: Analysis of the National Tuberculosis Registry 2000-2012. PloS One [Internet], 2015 [cited 2019 Dec 10]; 10(6):1-14. DOI: https://doi.org/10.1371/journal.pone.0129312

25. Pande T, Huddart S, Xavier W, Kulavalli, S, Chen T, Pai M, Saravu K. Prevalence of diabetes mellitus amongst hospitalized tuberculosis patients at an Indian tertiary care center: A descriptive analysis. PloS one [Internet], 2018 [cited 2020 Aug 13]: 13(7). DOI: https://doi.org/10.1371/journal.pone.0200838 
26. Workneh MH, Bjune GA, Yimer SA. Prevalence and Associated Factors of Diabetes Mellitus among Tuberculosis Patients in South-Eastern Amhara Region, Ethiopia: A Cross Sectional Study. PloS One [Internet], 2016 [cited 2019 Dec 10]; 11(1):1-15. DOI: https://doi.org/10.1371/journal.pone.0147621

27. Moreno-Martínez A, Casals M., Orcau À, Gorrindo P, Masdeu E, Cayla, JA. Factors associated with diabetes mellitus among adults with tuberculosis in a large European city, 2000-2013. Int. J. Tuberc. Lung. Dis. [Internet], 2015 [cited 2019 Dec 10]; 19 (12): 1507-12. DOI: https://doi.org/10.5588/ijtld.15.0102

28. Hongguang C, Min L, Shiwen J, Fanghui G, Shaoping H, Tiejie, G, et al. Impact of diabetes on clinical presentation and treatment outcome of pulmonary tuberculosis in Beijing. Epidemiology and Infection [Internet], 2015 [cited 2019 Dec 10]; 143 (1): 150-6. DOI: https://doi.org/10.1017/S095026881400079X

29. Herrera MT, Leiva ZE, Martín HF, Miranda OM, Morales OC. Associated tuberculosis and diabetes mellitus in Santiago de Chile Metropolitan area. Rev. chil. enferm. respir. [Internet], 2013 [cited 2019 Dec 10]; 29 (3): 171-5. DOI: http://dx.doi.org/10.4067/S0717-73482013000300008

30. Tatar D, Senol G, Alptekin S, Karakurum C, Aydin M, Coskunol I. Tuberculosis in diabetics: features in an endemic area. Jpn J. Infect. Dis. [Internet], 2009 [cited 2019 Dec 10]; 62(6): 423-7. DOI: https://pubmed.ncbi.nlm.nih.gov/19934532/

31. Badawi A, Sayegh S, Sallam M, Sadoun E, Al-Thani M, Alam MW, Arora P. The global relationship between the prevalence of diabetes mellitus and incidence of tuberculosis: 2000-2012. Glob. J. Health Sci. [Internet], 2014 [cited 2019 dez 04]; 7(2): 18391. DOI: http://dx.doi.org/10.5539/gjhs.v7n2p183

32. Al-Rifai RH, Pearson F, Critchley JA, Abu-Raddad LJ. Association between diabetes mellitus and active tuberculosis: A systematic review and meta-analysis. PLoS One [Internet], 2017 [cited 2019 Dec 10]; 12 (11): 1-26. DOI: https://doi.org/10.1371/journal.pone.0187967

33. Magee MJ, Bloss E, Shin SS, Contreras C, Huaman HA, Ticona JC, et al. Clinical characteristics, drug resistance, and treatment outcomes among tuberculosis patients with diabetes in Peru. Int. J Infect. Dis. [Internet], 2013 [cited 2019 Dec 10]; 17(6): 40412. DOI: https://doi.org/10.1371/journal.pone.0187967

34. Workneh MH, Bjune GA, Yimer SA. Prevalence and associated factors of tuberculosis and diabetes mellitus comorbidity: a systematic review. PloS One [Internet], 2017 [cited 2019 Dec 10]; 12(4):1-25. DOI: https://doi.org/10.1371/journal.pone.0175925

35. Lacerda SNB, Silva TD, Araújo PD, Pinto ML, Figueiredo TMRM. The comorbidity tuberculosis and diabetes mellitus. Rev. Enferm. UFPE [Internet], 2016 [cited 2019 Dec 10]; 10(1): 239-47. Avalaible from: https://periodicos.ufpe.br/revistas/revistaenfermagem/article/download/10946/12254

36. Macedo JL, Oliveira ASDSS, Pereira IC, Reis ER, Assunção MDJSM. Epidemiological profile of diabetes mellitus in northeastern Brazil. Res. Soc. Dev. [Internet], 2019 [cited 2019 Dec 10]; 8 (3):1-12. DOI: http://dx.doi.org/10.33448/rsd-v8i3.826

37. Kumar NP, Banurenkha VV, Nair D, Sridhar R, Kornfeld H, Nutman TB, Babu S. Coincident pre-diabetes is associated with dysregulated cytokine responses in pulmonary tuberculosis. PLoS One [Internet], 2014 [cited 2020 Jan 03]; (9): 1-8. DOI: https://doi.org/10.1371/journal.pone.0112108

38. Alavi SM, Khoshko MM, Salmanzadeh S, Eghtesad M. Comparison of Epidemiological, Clinical, Laboratory and Radiological Features of Hospitalized Diabetic and Non Diabetic Patients with pulmonary tuberculosis at Razi Hospital in Ahavaz Jundishapur. J. Microbial. [Internet], 2014 [cited 2020 Jan 03]; (7):1-5. DOI: https://dx.doi.org/10.5812\%2Fjjm.12447

39. Marques M, Cunha EAT, Evangelista MDSN, Basta PC, Marques AMC, Croda, J, Andrade SMO. Antituberculosis-drug resistance in the border of Brazil with Paraguay and Bolivia. Rev. Panam. Salud Publica [Internet], 2017 [cited 2019 Dec 10]; (41): 1-8. DOI: https://www.ncbi.nlm.nih.gov/pmc/articles/PMC6645181/\#

40. Zhou T, Liu X, Liu Y, Li X. Meta-analytic evaluation for the spatio-temporal patterns of the associations between common risk factors and type 2 diabetes in mainland China. Medicine [Internet], 2019 [cited 2019 Dec 10]; 98(20): 1-12. DOI: https://doi.org/10.1097/MD.0000000000015581 